\title{
HYPERASYMPTOTIC SOLUTIONS FOR CERTAIN PARTIAL DIFFERENTIAL EQUATIONS
}

\author{
SŁAWOMIR MICHALIK AND MARIA SUWIŃSKA
}

\begin{abstract}
We present the hyperasymptotic expansions for a certain group of solutions of the heat equation. We extend this result to a more general case of linear PDEs with constant coefficients. The generalisation is based on the method of Borel summability, which allows us to find integral representations of solutions for such PDEs.
\end{abstract}

\section{INTRODUCTION}

Errors generated in the process of estimating functions by a finite number of terms of their asymptotic expansions usually are of the form $\exp (-q / t)$ with $t \rightarrow 0$ and usually such a result is satisfactory. However, it is possible to obtain a refined information by means of finding the hyperasymptotic expansion of a given function, which amounts to expanding remainders of asymptotic expansions repeatedly.

More precisely, let us find the asymptotic expansion of a given function $F$. We receive

$$
F(t)=A_{0}+A_{1}+\ldots \text { for } t \rightarrow 0
$$

with $A_{i}=a_{i} t^{i}$. Once we truncate $(1)$ after a certain amount of terms, we receive an approximation of $F$ and

$$
F(t)=A_{0}+A_{1}+\ldots+A_{N_{0}-1}+R_{N_{0}}(t) .
$$

The optimal value of $N_{0}=N_{0}(t)$ can be found by means of minimization of the remainder $R_{N_{0}}(t)$. After that we consider $R_{N_{0}}(t)$ as a function of two variables $t$ and $N_{0}$ and expand it in a new asymptotic series

$$
R_{N_{0}}(t)=B_{0}+B_{1}+\ldots,
$$

which can be truncated optimally after $N_{1}$ terms. Thus we receive an estimation of $F$ of the form

$$
F(t)=A_{0}+A_{1} \ldots+A_{N_{0}-1}+B_{0}+B_{1}+\ldots+B_{N_{1}-1}+R_{N_{1}}(t)
$$

and the remainder $R_{N_{1}}(t)$ appears to be exponentially small compared to $R_{N_{0}}(t)$.

After repeating the process $n$ times we receive the $n$-th level hyperasymptotic expansion of $F$ as $t \rightarrow 0$ :

$F(t)=A_{0}+\ldots+A_{N_{0}-1}+B_{0}+\ldots+B_{N_{1}-1}+C_{0}+\ldots C_{N_{2}-1}+\ldots+R_{N_{n}}(t)$.

2010 Mathematics Subject Classification. 35C20, 35G10, 35K05.

Key words and phrases. hyperasymptotic expansions, heat equation, linear PDEs with constant coefficients, $k$-summability. 
The concept of hyperasymptotic expansions emerged in 1990 as a topic of an article by M. V. Berry and C. J. Howls [2] and it was conceived as a way to estimate the solutions of Schrödinger-type equations. Methods of obtaining hyperasymptotic expansions were then developed mostly by A. B. Olde Daalhuis, who found an expansion for the confluent hypergeometric function [7, 8], linear ODEs with the singularity of rank one [9] and various nonlinear ODEs [10, 11].

Using the results from [7] and [8], we will find a hyperasymptotic expansion for a certain group of solutions of the heat equation. To this end we will first obtain the optimal number of terms, after which the asymptotic expansion of the solution should be truncated. This will enable us to estimate the remainder using the Laplace method (see [12]). The reasoning then will be adapted to the case of $n$-level hyperasymptotic expansion.

Our main goal is to generalise those results to the case of linear PDEs with constant coefficients. To this end, first we reduce the general linear PDEs in two variables with constant coefficients to simple pseudodifferential equations using the methods of [4, 5]. Next, we apply the theory of summability, which allows to construct integral representations of solutions of such equations. Finally, in a similar way to the heat equation, we construct hyperasymptotic expansions for such integral representations of solutions.

Throughout the paper the following notation will be used.

A sector $S$ in a direction $d \in \mathbb{R}$ with an opening $\alpha>0$ in the universal covering space $\tilde{\mathbb{C}}$ of $\mathbb{C} \backslash\{0\}$ is defined by $S_{d}(\alpha):=\left\{z \in \tilde{\mathbb{C}}: z=r e^{i \varphi}, r>\right.$ $0, \varphi \in(d-\alpha / 2, d+\alpha / 2)\}$. If the opening $\alpha$ is not essential, the sector $S_{d}(\alpha)$ is denoted briefly by $S_{d}$.

We denote by $D_{r}$ a complex disc in $\mathbb{C}$ with radius $r>0$ and the center in 0 , i.e. $D_{r}:=\{z \in \mathbb{C}:|z|<r\}$. In case that the radius $r$ is not essential, the set $D_{r}$ will be designated briefly by $D$.

If a function $f$ is holomorphic on a domain $G \subset \mathbb{C}^{n}$, then it will be denoted by $f \in \mathcal{O}(G)$. Analogously, the space of holomorphic functions on a domain $G \subset \mathbb{C}^{n}$ with respect to the variable $z^{1 / \gamma}:=z_{1}^{1 / \gamma_{1}}, \ldots, z_{n}^{1 / \gamma_{n}}$, where $1 / \gamma:=$ $\left(1 / \gamma_{1}, \ldots, 1 / \gamma_{n}\right)$ and $\left(\gamma_{1}, \ldots, \gamma_{n}\right) \in \mathbb{N}^{n}$, is denoted by $\mathcal{O}_{1 / \gamma}(G)$.

By $\partial G$ we mean the boundary of the set $G$.

\section{HYPERASYMPTOTIC EXPANSIONS FOR THE HEAT EQUATION}

Let us consider the Cauchy problem for the heat equation

$$
\left\{\begin{aligned}
u_{t}(t, z)-u_{z z}(t, z) & =0 \\
u(0, z) & =\varphi(z) .
\end{aligned}\right.
$$

We assume that the function $\varphi$ has finitely many isolated singular points (singlevalued and branching points) on $\mathbb{C}$. Without loss of generality we may assume 
that the set of singular points of $\varphi$ is given by

$$
\begin{array}{r}
\mathcal{A}:=\left\{a_{i j} \in \mathbb{C}: \arg \left(a_{i 1}\right)=\cdots=\arg \left(a_{i L_{i}}\right)=\lambda_{i},\left|a_{i 1}\right|<\left|a_{i 2}\right|<\ldots<\left|a_{i L_{i}}\right|,\right. \\
\left.j=1, \ldots, L_{i}, i=1, \ldots, K\right\},
\end{array}
$$

where $K \in \mathbb{N}, L_{1}, \ldots, L_{K} \in \mathbb{N}$ and $\lambda_{1}, \ldots, \lambda_{K} \in \mathbb{R}$ satisfy $\lambda_{1}<\cdots<\lambda_{K}$.

Under these conditions we can define the set $H$ as a sum of a finite number of half-lines (see Figure 1 , such that $H:=\bigcup_{i=1}^{K}\left\{a_{i 1} t: t \geq 1\right\}$. So we may assume that $\varphi \in \mathcal{O}(\mathbb{C} \backslash H)$ and $\mathcal{A}$ is the set of all singular points of $\varphi$. We denote it briefly by $\varphi \in \mathcal{O}_{\mathcal{A}}(\mathbb{C} \backslash H)$.

Moreover, let us assume that for any $\xi>0$ there exist positive constants $B$ and $C$ such that $|\varphi(z)| \leq C e^{B|z|^{2}}$ for all $z \in \mathbb{C} \backslash H_{\xi}$, where $H_{\xi}:=\{z \in$ $\mathbb{C}$ : dist $(z, H)<\xi\}$. We write it $\varphi \in \mathcal{O}_{\mathcal{A}}^{2}(\mathbb{C} \backslash H)$ for short.

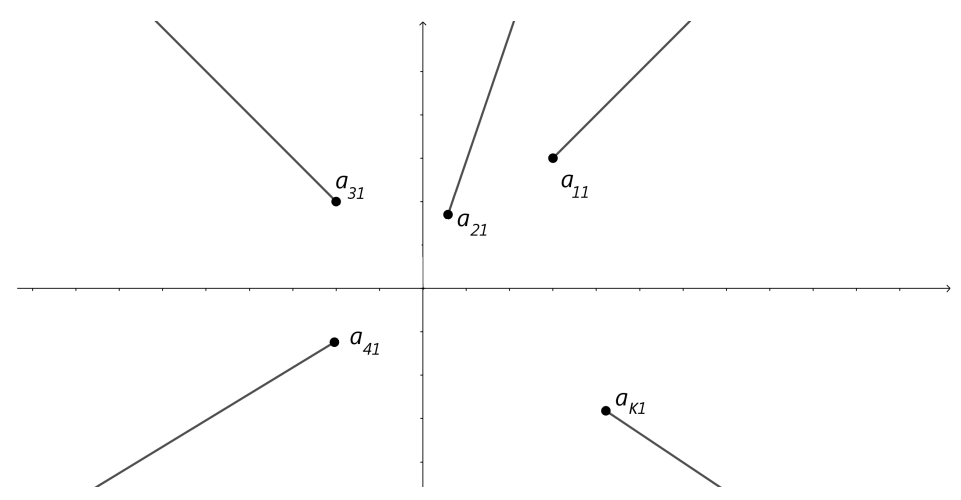

FIGURE 1.

The solution of (2) is given by (see [6, Theorem 4])

$$
u(t, z)=\frac{1}{2 \sqrt{\pi t}} \int_{e^{i \frac{\theta}{2}} \mathbb{R}} e^{-\frac{s^{2}}{4 t}} \varphi(z+s) d s
$$

under condition that $\theta$ is not the Stokes line for $u$ (see [6, Definition 7], i.e. $\theta \neq 2 \lambda_{i} \bmod 2 \pi$ for $i=1, \ldots, K$.

To separate from the Stokes lines we fix a small positive number $\delta$ and we assume that

$$
\left|\left(\theta-2 \lambda_{i}\right) \quad \bmod 2 \pi\right| \geq \delta \quad \text { for all } \quad i=1, \ldots, K .
$$

In other words we assume that $\theta \in[0,2 \pi) \backslash \bigcup_{i=1}^{K}\left(2 \lambda_{i}-\delta, 2 \lambda_{i}+\delta\right) \bmod 2 \pi$.

Our goal is to find a hyperasymptotic expansion of (3) for $t \rightarrow 0$ and $\arg t=\theta$ with $z$ belonging to a small neighbourhood of 0 . To this end we fix a sufficiently small constant $\tilde{\varepsilon}$ such that $\varphi(z) \in \mathcal{O}\left(D_{\tilde{\varepsilon}}\right)$. 
2.1. 0-level hyperasymptotic expansion. To find the hyperasymptotic expansion of the solution of (2) we will use the method described in [7] (see also [8]) in the case of the confluent hypergeometric functions. In order to do so let us modify the right-hand side of (3) by

$$
u(t, z)=\frac{1}{4 \sqrt{\pi t}} \int_{0}^{e^{i \theta} \infty} e^{-\frac{s}{4 t}} s^{-\frac{1}{2}}\left[\varphi\left(z+s^{\frac{1}{2}}\right)+\varphi\left(z-s^{\frac{1}{2}}\right)\right] d s .
$$

Replacing $s$ and $t$ by $|s| e^{i \theta}$ and $|t| e^{i \theta}$, respectively, we obtain

$$
u(t, z)=\frac{1}{2 \sqrt{\pi|t|}} \int_{0}^{\infty} \frac{e^{-\frac{s}{4|t|}}}{2 \sqrt{s}}\left[\varphi\left(z+e^{i \frac{\theta}{2}} \sqrt{s}\right)+\varphi\left(z-e^{i \frac{\theta}{2}} \sqrt{s}\right)\right] d s .
$$

To find the asymptotic expansion of (5), we will expand the function

$$
f_{0}(s, z):=\frac{1}{2}\left[\varphi\left(z+e^{i \frac{\theta}{2}} \sqrt{s}\right)+\varphi\left(z-e^{i \frac{\theta}{2}} \sqrt{s}\right)\right]
$$

around the point $s=0$ using the complex Taylor formula. We receive

$$
f_{0}(s, z)=\sum_{k=0}^{N_{0}-1} \frac{\varphi^{(2 k)}(z)}{(2 k) !} e^{i k \theta} s^{k}+f_{1}(s, z) s^{N_{0}}
$$

where $f_{1}(s, z)$ is of the form

$$
f_{1}(s, z):=\frac{1}{2 \pi i} \int_{\Omega_{0}(0, s)} \frac{f_{0}(w, z)}{w^{N_{0}}(w-s)} d w
$$

and the contour $\Omega_{0}(0, s)$ is a boundary of the sum of two discs such that all singular points of $f_{0}(w, z)$ are located outside of those discs and points 0 and $s$ are both inside. More precisely, let us take $r:=\min _{1 \leq i \leq K}\left|a_{i 1}\right|-\tilde{\varepsilon}$. In this case we can put $\Omega_{0}(0, s)$ as

$$
\Omega_{0}(0, s):=\partial\left(\left\{w \in \mathbb{C}:|w| \leq r^{2}-\varepsilon\right\} \cup\left\{w \in \mathbb{C}:|w-s| \leq \frac{\varepsilon}{2}\right\}\right)
$$

for some $\varepsilon \in\left(0, r^{2} / 2\right)$ and $\varepsilon$ separate from 0 . It is possible to take such a contour, because by (4) we may choose so small $\tilde{\varepsilon}>0$ that for $z \in D_{\tilde{\varepsilon}}$ the singularities $w_{i j}(z):=\left(a_{i j}-z\right)^{2} e^{-i \theta}$ of $f_{0}(w, z)$ will never be positive real numbers. So we are able to choose $\varepsilon$ satisfying additionally

$$
\varepsilon<\frac{1}{2} \inf _{z \in D_{\tilde{\varepsilon}}} \min _{\substack{i=1, \ldots K \\ j=1, \ldots, L_{i}}} \operatorname{dist}\left(w_{i j}(z), \mathbb{R}_{+}\right)
$$

and then $\Omega_{0}(0, s)$ satisfies the desired conditions.

Using (6) and basic properties of the gamma function, we can obtain an expansion of (5) of the form

$$
u(t, z)=\sum_{k=0}^{N_{0}-1} \frac{\varphi^{(2 k)}(z)}{k !} t^{k}+R_{N_{0}}(t, z)
$$


where

$$
R_{N_{0}}(t, z)=\frac{1}{2 \sqrt{\pi|t|}} \int_{0}^{\infty} e^{-\frac{s}{4|t|}} s^{N_{0}-\frac{1}{2}} f_{1}(s, z) d s .
$$

Seeing as $|w| \geq r^{2}-\varepsilon,|w-s| \geq \frac{\varepsilon}{2}$ and assuming that all the conditions given for the Cauchy datum hold, we can find the optimal value of $N_{0}=N_{0}(t)$. The first step to do so is finding an estimation of $f_{1}(s, z)$. Let us note that there exist positive constants $\tilde{A}$ and $\tilde{B}$ such that $\left|\varphi\left(z \pm e^{i \frac{\theta}{2}} \sqrt{w}\right)\right| \leq \tilde{A} e^{\tilde{B} s}$ for any $w \in \Omega_{0}(0, s), s>0$ and $z \in D_{\tilde{\varepsilon}}$. Hence

$$
\begin{aligned}
\left|f_{1}(s, z)\right| \leq \frac{1}{2 \pi} \int_{\Omega_{0}(0, s)} \frac{2 \tilde{A} e^{\tilde{B} s}}{\varepsilon\left(r^{2}-\varepsilon\right)^{N_{0}}} d|w| \leq & \frac{2 \tilde{A} e^{\tilde{B} s}}{\varepsilon\left(r^{2}-\varepsilon\right)^{N_{0}}}\left(r^{2}-\varepsilon+\frac{\varepsilon}{2}\right) \\
& \leq \frac{2 \tilde{A} r^{2} e^{\tilde{B} s}}{\varepsilon\left(r^{2}-\varepsilon\right)^{N_{0}}}=\frac{A_{0} e^{\tilde{B} s}}{\left(r^{2}-\varepsilon\right)^{N_{0}}}
\end{aligned}
$$

for $A_{0}:=2 \tilde{A} r^{2} / \varepsilon$. As a consequence,

$$
\left|R_{N_{0}}(t, z)\right| \leq \frac{A_{0}}{2 \sqrt{\pi|t|}} \int_{0}^{\infty} e^{-\frac{s}{4|t|}} s^{N_{0}-\frac{1}{2}}\left(r^{2}-\varepsilon\right)^{-N_{0}} e^{\tilde{B} s} d s .
$$

It is easy to check that the integrand of (10) has a maximum at a certain point $s=\sigma_{1}$ which satisfies the condition $N_{0}=\sigma_{1}\left(\frac{1}{4|t|}-\tilde{B}\right)+\frac{1}{2}$, and so now we can find the point where the minimum with respect to $\sigma_{1}$ of the function given by the formula

$$
\sigma_{1} \mapsto e^{-\sigma_{1}\left(\frac{1}{4|t|}-\tilde{B}\right)} \sigma_{1}^{\sigma_{1}\left(\frac{1}{4|t|}-\tilde{B}\right)}\left(r^{2}-\varepsilon\right)^{-\sigma_{1}\left(\frac{1}{4|t|}-\tilde{B}\right)-\frac{1}{2}}
$$

is attained. This function is minimal at $\sigma_{1}=r^{2}-\varepsilon$. Because of these facts we can choose the optimal $N_{0}:=\left\lfloor\left(r^{2}-\varepsilon\right)\left(\frac{1}{4|t|}-\tilde{B}\right)+\frac{1}{2}\right\rfloor$, where by $\lfloor\cdot\rfloor$ we denote the integer part of a real number. Next, we take $\sigma_{1}:=\frac{N_{0}-\frac{1}{2}}{\frac{1}{4|t|}-\tilde{B}}$. Thus $\sigma_{1} \leq r^{2}-\varepsilon$.

Thanks to that we are able to use the Laplace method, described at length in [12], and to estimate the right-hand side of (10). So, we conclude that

$$
\left|R_{N_{0}}(t, z)\right| \sim O\left(\frac{e^{-\sigma_{1}\left(\frac{1}{4|t|}-\tilde{B}\right)}}{\sqrt{1-4 \tilde{B}|t|}}\right) \quad \text { for } \quad t \rightarrow 0, \quad \arg t=\theta .
$$

2.2. n-level hyperasymptotic expansion. When the $n$-level asymptotic expansion is known, it is easy to compute the $(n+1)$-level expansion using the method presented in Section 2.1. 
Observe that the remainder obtained in the $n$-level hyperasymptotic expansion is of the form

$$
R_{N_{n}}(t, z)=\frac{1}{2 \sqrt{\pi|t|}} \int_{0}^{\infty} e^{-\frac{s}{4|t|}} s^{N_{0}-\frac{1}{2}}\left(s-\sigma_{1}\right)^{N_{1}} \cdot \ldots \cdot\left(s-\sigma_{n}\right)^{N_{n}} f_{n+1}(s, z) d s
$$

where

and

$$
f_{n+1}(s, z):=\frac{1}{2 \pi i} \int_{\Omega_{n}\left(\sigma_{n}, s\right)} \frac{f_{n}(w, z)}{\left(w-\sigma_{n}\right)^{N_{n}}(w-s)} d w
$$

$\Omega_{n}\left(\sigma_{n}, s\right):=\partial\left(\left\{w \in \mathbb{C}:\left|w-\sigma_{n}\right| \leq d\left(\sigma_{n}, \theta\right)-\rho_{n} \varepsilon\right\} \cup\left\{w \in \mathbb{C}:|w-s| \leq 2^{-n-1} \varepsilon\right\}\right)$

with $d(w, \theta):=\inf _{z \in D_{\tilde{\varepsilon}}} \inf _{\zeta \in H}\left|w-e^{-i \theta}(z-\zeta)^{2}\right|$ and $\rho_{n}:=2-2^{-n}$. The contour is chosen in this way so that, when we express $f_{n+1}$ in terms of $f_{0}$, that is as a multiple integral of the form

$$
\begin{aligned}
f_{n+1}(s, z)= & \frac{1}{(2 \pi i)^{n+1}} \int_{\Omega_{n}\left(\sigma_{n}, s\right)} \int_{\Omega_{n-1}\left(\sigma_{n-1}, x_{n}\right)} \ldots \int_{\Omega_{0}\left(0, x_{1}\right)} f_{0}\left(x_{0}, z\right) . \\
& \cdot \frac{1}{x_{0}^{N_{0}} \prod_{k=1}^{n}\left[\left(x_{k}-\sigma_{k}\right)^{N_{k}}\left(x_{k-1}-x_{k}\right)\right]\left(x_{n}-s\right)} d x_{0} \ldots d x_{n} .
\end{aligned}
$$

We show that all the singular points of $x_{0} \mapsto f_{0}\left(x_{0}, z\right)$ are outside of the area surrounded by $\Omega_{n}\left(\sigma_{n}, s\right), \Omega_{n-1}\left(\sigma_{n-1}, x_{n}\right), \ldots, \Omega_{0}\left(0, x_{1}\right)$. To this end we take $x_{k} \in \Omega_{k}\left(\sigma_{k}, x_{k+1}\right)$ for $k=0, \ldots, n$ with the notation $\sigma_{0}:=0$ and $x_{n+1}:=s$. It is sufficient to prove that $d\left(x_{0}, \theta\right) \geq \varepsilon$. There are two possibilities.

In the first case $\left|x_{k}-x_{k-1}\right|=2^{-k-1} \varepsilon$ for $k=1, \ldots, n$. Then

$$
\left|x_{0}-s\right| \leq \sum_{k=0}^{n}\left|x_{k+1}-x_{k}\right| \leq \sum_{k=0}^{n} 2^{-k-1} \varepsilon=\left(1-2^{-n-1}\right) \varepsilon
$$

Since $d(s, \theta) \geq 2 \varepsilon$ we get $d\left(x_{0}, \theta\right) \geq d(s, \theta)-\left|x_{0}-s\right| \geq \varepsilon$.

In the second case there exists $m \in\{1, \ldots, n\}$ such that $\left|x_{k}-x_{k-1}\right|=2^{-k-1} \varepsilon$ for $k=1, \ldots, m-1$ and $\left|x_{m}-\sigma_{m}\right|=d\left(\sigma_{m}, \theta\right)-\rho_{m} \varepsilon$. Hence $\left|x_{0}-x_{m}\right| \leq$ $\left(1-2^{-m}\right) \varepsilon$ and $d\left(x_{m}, \theta\right) \geq d\left(\sigma_{m}, \theta\right)-\left|x_{m}-\sigma_{m}\right| \geq \rho_{m} \varepsilon$, so we conclude that $d\left(x_{0}, \theta\right) \geq d\left(x_{m}, \theta\right)-\left|x_{0}-x_{m}\right| \geq \varepsilon$.

Using the same algorithm as in the case of the 0-level expansion, we can estimate $R_{N_{n}}(t, z)$ as follows

$$
\begin{gathered}
\left|R_{N_{n}}(t, z)\right| \leq \frac{A_{n}}{2 \sqrt{\pi|t|}} \int_{0}^{\infty} e^{-\frac{s}{4|t|}+\tilde{B} s} \frac{s^{N_{0}-\frac{1}{2}}}{\left(r^{2}-\varepsilon\right)^{N_{0}}} \cdot \frac{\left|s-\sigma_{1}\right|^{N_{1}}}{\left(d\left(\sigma_{1}, \theta\right)-\rho_{1} \varepsilon\right)^{N_{1}}} \cdot \ldots \cdot \\
\cdot \frac{\left|s-\sigma_{n}\right|^{N_{n}}}{\left(d\left(\sigma_{n}, \theta\right)-\rho_{n} \varepsilon\right)^{N_{n}}} d s
\end{gathered}
$$

for a certain constant $A_{n}$.

Next, we find points where the integrand on the right-hand side of (12) attains its local maxima. Let us observe that this function has $n+1$ maxima in points 
$s_{1}, \ldots, s_{n+1}$ such that $s_{1}<\sigma_{1}<s_{2}<\ldots<\sigma_{n}<s_{n+1}$ and all $s_{j}$ satisfy the condition:

$$
\frac{1}{4|t|}-\tilde{B}=\frac{N_{0}-\frac{1}{2}}{s_{j}}+\frac{N_{1}}{s_{j}-\sigma_{1}}+\ldots+\frac{N_{n}}{s_{j}-\sigma_{n}} .
$$

From (13) we conclude that $s_{j}$ are decreasing functions of $N_{n}$ for $1 \leq j \leq n$ and $s_{n+1}$ increases with respect to $N_{n}$. Moreover, the value of the integrand in (12) in the points $s_{j}, 1 \leq j \leq n$, decreases with respect to $N_{n}$. However, it behaves differently in the point $s_{n+1}$. It decreases with respect to $N_{n}$ when

$$
\frac{s_{n+1}-\sigma_{n}}{d\left(\sigma_{n}, \theta\right)-\rho_{n} \varepsilon}<1
$$

that is for $s_{n+1} \in\left(\sigma_{n}, \sigma_{n}+d\left(\sigma_{n}, \theta\right)-\rho_{n} \varepsilon\right)$, and increases when $s_{n+1} \in\left(\sigma_{n}+\right.$ $\left.d\left(\sigma_{n}, \theta\right)-\rho_{n} \varepsilon,+\infty\right)$. Hence there exist $N_{n} \in \mathbb{N}$ and $s_{n+1}=: \sigma_{n+1}$ satisfying (13) for which the integrand reaches its minimal value (see [8]).

Again, we can use the Laplace method to obtain the estimation of $R_{N_{n}}$ (compare [7] and [8])

$$
\left|R_{N_{n}}(t, z)\right| \sim O\left(\frac{e^{-\eta_{n}\left(\frac{1}{4|t|}-\tilde{B}\right)}}{\sqrt{1-4 \tilde{B}|t|}}\right) \quad \text { for } \quad t \rightarrow 0, \quad \arg t=\theta .
$$

We have the sequence of positive numbers $\eta_{0}=\sigma_{1} \sim r^{2}<\eta_{1}<\eta_{2}<\eta_{3}<\ldots$, but it is not clear, whether or not, $\left\{\eta_{n}\right\}_{n \in \mathbb{N}}$ is an unbounded sequence (see [7] and [8]).

To find the $(n+1)$-level hyperasymptotic expansion we expand the function $s \mapsto f_{n+1}(s, z)$ around the point $\sigma_{n+1}$. As a result we receive a series

$$
f_{n+1}(s, z)=\sum_{j=0}^{N_{n+1}-1} b_{n+1, j}(z)\left(s-\sigma_{n+1}\right)^{j}+\left(s-\sigma_{n+1}\right)^{N_{n+1}} f_{n+2}(s, z),
$$

which, after substituting it in (11), gives us the $(n+1)$-level expansion of the form

$$
\begin{array}{r}
R_{N_{n}}(t, z)=\frac{1}{\sqrt{\pi|t|}} \sum_{j=0}^{N_{n+1}-1} b_{n+1, j}(z) \int_{0}^{\infty} \frac{e^{-\frac{s}{4|t|}} s^{N_{0}}}{2 \sqrt{s}}\left(s-\sigma_{1}\right)^{N_{1}} \cdot \ldots \cdot \\
\cdot\left(s-\sigma_{n}\right)^{N_{n}}\left(s-\sigma_{n+1}\right)^{j} d s+R_{N_{n+1}}(t, z) .
\end{array}
$$

Moreover, since

$$
s^{N_{0}}\left(s-\sigma_{1}\right)^{N_{1}} \cdots\left(s-\sigma_{n}\right)^{N_{n}}\left(s-\sigma_{n+1}\right)^{j}=\sum_{l=0}^{N_{0}+\cdots+N_{n}+j} a_{n, j, l} s^{l}
$$

is a polynomial of degree $N_{0}+\cdots+N_{n}+j$, and by the properties of the gamma function

$$
\frac{1}{\sqrt{\pi|t|}} \int_{0}^{\infty} \frac{e^{-\frac{s}{4|t|}}}{2 \sqrt{s}} s^{l} d s=\frac{(2 l) !}{l !}|t|^{l}=\frac{(2 l) !}{l !} e^{-i \theta l} t^{l} \quad \text { for } \quad l=0,1, \ldots,
$$


we conclude that

$$
R_{N_{n}}(t, z)=\sum_{j=0}^{N_{n+1}-1} b_{n+1, j}(z) \sum_{l=0}^{N_{0}+\cdots+N_{n}+j} \frac{(2 l) !}{l !} a_{n, j, l} e^{-i \theta l} t^{l}+R_{N_{n+1}}(t, z) .
$$

Hence the hyperasymptotic expansion of $u$ takes the form

$$
u(t, z)=\sum_{l=0}^{N_{0}+\cdots+N_{n}-1} \psi_{l}(z) t^{l}+R_{N_{n}}(t, z)
$$

for some functions $\psi_{l}(z)$ depending on $b_{n+1, j}(z)$ and $a_{n, j, l}$.

2.3. Conclusion. We can formulate the following theorem regarding the hyperasymptotic expansion of (2)

Theorem 1. For any $n \in \mathbb{N}$ the solution (3) of the heat equation has the hyperasymptotic expansion as $t \rightarrow 0$ in the direction $\theta \in[0,2 \pi) \backslash \bigcup_{i=1}^{K}\left(2 \lambda_{i}-\delta, 2 \lambda_{i}+\delta\right)$ $\bmod 2 \pi$ of the form

$$
\begin{gathered}
u(t, z)=\sum_{j=0}^{N_{0}-1} \frac{\varphi^{(2 j)}(z)}{j !} t^{j}+\sum_{m=1}^{n} \sum_{j=0}^{N_{m}-1} \frac{b_{m, j}(z)}{\sqrt{\pi|t|}} \int_{0}^{\infty} \frac{e^{-\frac{s}{4|t|} s^{N_{0}}}}{2 \sqrt{s}}\left(s-\sigma_{1}\right)^{N_{1}} \\
\cdots\left(s-\sigma_{m-1}\right)^{N_{m-1}}\left(s-\sigma_{m}\right)^{j} d s+R_{N_{n}}(t, z)=\sum_{l=0}^{N_{0}+\cdots+N_{n}-1} \psi_{l}(z) t^{l}+R_{N_{n}}(t, z),
\end{gathered}
$$

where the remainder $R_{N_{n}}(t, z)$ is of the form

$$
R_{N_{n}}(t, z)=\frac{1}{2 \sqrt{\pi|t|}} \int_{0}^{\infty} e^{-\frac{s}{4|t|}} s^{N_{0}-\frac{1}{2}}\left(s-\sigma_{1}\right)^{N_{1}} \cdot \ldots \cdot\left(s-\sigma_{n}\right)^{N_{n}} f_{n+1}(s, z) d s
$$

and for any $m \leq n$ and $j<N_{m}$

$$
\begin{aligned}
& f_{m}(s, z)=\frac{1}{(2 \pi i)^{m}} \int_{\Omega_{m-1}\left(\sigma_{m-1}, s\right)} \int_{\Omega_{m-2}\left(\sigma_{m-2}, x_{m-1}\right)} \cdots \int_{\Omega_{1}\left(\sigma_{1}, x_{2}\right)} \int_{\Omega_{0}\left(0, x_{1}\right)} \\
& \frac{f_{0}\left(x_{0}, z\right) d x_{0} \ldots d x_{m-1}}{x_{0}^{N_{0}} \prod_{k=1}^{m-1}\left[\left(x_{k}-\sigma_{k}\right)^{N_{k}}\left(x_{k-1}-x_{k}\right)\right]\left(x_{m-1}-s\right)}
\end{aligned}
$$

and

Moreover,

$$
b_{m, j}(z)=\left.\frac{1}{j !} \frac{\partial^{j}}{\partial s^{j}} f_{m}(s, z)\right|_{s=\sigma_{m}}
$$

$$
\left|R_{N_{n}}(t, z)\right| \sim O\left(\frac{e^{-\eta_{n}\left(\frac{1}{4|t|}-\tilde{B}\right)}}{\sqrt{1-4 \tilde{B}|t|}}\right) \quad \text { as } \quad t \rightarrow 0, \arg t=\theta, z \in D_{\tilde{\varepsilon}}
$$

for some sequence of positive numbers $\eta_{0}=\sigma_{1} \sim r^{2}<\eta_{1}<\eta_{2}<\eta_{3}<\ldots$ 


\section{Generalisation to Linear PDEs With CONSTANT COEFFICIENTS}

In this section we show how to find the hyperasymptotic expansion for solutions of general linear non-Cauchy-Kowalevskaya type PDEs with constant coefficients. The result is based on the theory of summability which allows us to construct the actual solution, which is analytic in some sectorial neighbourhood of the origin, from the divergent formal power series solution. Moreover this actual solution has an integral representation in the similar form to (3).

3.1. Summability. First, we define $k$-summability in a similar way to [6]. For more information about the theory of summability we refer the reader to [1].

We say that a formal power series $\hat{u}(t, z)=\sum_{n=0}^{\infty} \frac{u_{n}(z)}{n !} t^{n}$ with $u_{n}(z) \in \mathcal{O}_{1 / \kappa}(D)$ is a Gevrey series of order $q$ if there exist $A, B, r>0$ such that $\left|u_{n}(z)\right| \leq$ $A B^{n}(n !)^{q+1}$ for every $|z|<r$ and every $n \in \mathbb{N}$. We denote by $\mathcal{O}_{1 / \kappa}(D)[[t]]_{q}$ the set of such formal power series.

Moreover, for $k>0$ and $d \in \mathbb{R}$, we say that $\hat{u}(t, z) \in \mathcal{O}_{1 / \kappa}(D)[[t]]_{\frac{1}{k}}$ is $k$ summable in a direction $d$ if its $k$-Borel transform

$$
v(s, z):=\left(\mathcal{B}_{k} \hat{u}\right)(s, z):=\sum_{n=0}^{\infty} \frac{u_{n}(z)}{\Gamma(1+(1+1 / k) n)} s^{n},
$$

where $\Gamma(\cdot)$ denotes the gamma function, is analytically continued with respect to $s$ to an unbounded sector $S_{d}$ in a direction $d$ and this analytic continuation has exponential growth of order $k$ as $s$ tends to infinity (i.e. $|v(s, z)| \leq A e^{B|s|^{k}}$ as $s \rightarrow \infty)$. We denote it briefly by $v(s, z) \in \mathcal{O}_{1,1 / \kappa}^{k}\left(\left(D \cup S_{d}\right) \times D\right)$. In this case the $k$-sum of $\hat{u}(t, z)$ in the direction $d$ is given by

$$
u^{d}(t, z):=\left(\mathcal{L}_{k, d} v\right)(t, z):=t^{-k /(1+k)} \int_{e^{i d \mathbb{R}_{+}}} v(s, z) C_{(k+1) / k}\left((s / t)^{\frac{k}{1+k}}\right) d s^{\frac{k}{1+k}},
$$

where $C_{\alpha}(\tau)$ is the Ecalle kernel defined by

$$
C_{\alpha}(\tau):=\sum_{n=0}^{\infty} \frac{(-\tau)^{n}}{n ! \Gamma\left(1-\frac{n+1}{\alpha}\right)} .
$$

3.2. Reduction of linear PDEs with constant coefficients to simple pseudodifferential equations. We consider the Cauchy problem

$$
\left\{\begin{array}{l}
P\left(\partial_{t}, \partial_{z}\right) u=0 \\
\partial_{t}^{j} u(0, z)=\varphi_{j}(z) \in \mathcal{O}_{\mathcal{A}}(\mathbb{C} \backslash H)
\end{array}\right.
$$

where $P(\lambda, \zeta):=P_{0}(\zeta) \lambda^{m}-\sum_{j=1}^{m} P_{j}(\zeta) \lambda^{m-j}$ is a general polynomial of two variables, which is of order $m$ with respect to $\lambda$.

First, we show how to use the methods from [4, 5] for the reduction of (17) to simple pseudodifferential equations. 
If $P_{0}(\zeta)$ is not a constant, then a formal solution of $(17)$ is not uniquely determined. To avoid this inconvenience we choose some special solution which is already uniquely determined. To this end we factorise the polynomial $P(\lambda, \zeta)$ as follows

$$
P(\lambda, \zeta)=P_{0}(\zeta)\left(\lambda-\lambda_{1}(\zeta)\right)^{m_{1}} \cdots\left(\lambda-\lambda_{l}(\zeta)\right)^{m_{l}}=: P_{0}(\zeta) \widetilde{P}(\lambda, \zeta)
$$

where $\lambda_{1}(\zeta), \ldots, \lambda_{l}(\zeta)$ are the roots of the characteristic equation $P(\lambda, \zeta)=0$ with multiplicity $m_{1}, \ldots, m_{l}\left(m_{1}+\cdots+m_{l}=m\right)$ respectively.

Since $\lambda_{\alpha}(\zeta)$ are algebraic functions, we may assume that there exist $\kappa \in \mathbb{N}$ and $r_{0}<\infty$ such that $\lambda_{\alpha}(\zeta)$ are holomorphic functions of the variable $\xi=\zeta^{1 / \kappa}$ (for $|\zeta| \geq r_{0}$ and $\alpha=1, \ldots, l$ ) and, moreover, there exist $\lambda_{\alpha} \in \mathbb{C} \backslash\{0\}$ and $q_{\alpha}=\mu_{\alpha} / \nu_{\alpha}$ (for some relatively prime numbers $\mu_{\alpha} \in \mathbb{Z}$ and $\nu_{\alpha} \in \mathbb{N}$ ) such that $\lambda_{\alpha}(\zeta) \sim \lambda_{\alpha} \zeta^{q_{\alpha}}$ for $\alpha=1, \ldots, l$ (i.e. $\lim _{\zeta \rightarrow \infty} \frac{\lambda_{\alpha}(\zeta)}{\zeta^{q_{\alpha}}}=\lambda_{\alpha}, \lambda_{\alpha}$ and $q_{\alpha}$ are called respectively a leading term and a pole order of $\left.\lambda_{\alpha}(\zeta)\right)$. Observe that $\nu_{\alpha} \mid \kappa$ for $\alpha=1, \ldots, l$.

Following [5, Definition 13] we define the pseudodifferential operators $\lambda_{\alpha}\left(\partial_{z}\right)$ as

$$
\lambda_{\alpha}\left(\partial_{z}\right) \varphi(z):=\frac{1}{2 \kappa \pi i} \oint_{|w|=\varepsilon}^{\kappa} \varphi(w) \int_{e^{i \theta} r_{0}}^{e^{i \theta} \infty} \lambda_{\alpha}(\zeta) \mathbf{E}_{1 / \kappa}\left(\zeta^{1 / \kappa} z^{1 / \kappa}\right) e^{-\zeta w} d \zeta d w
$$

for every $\varphi \in \mathcal{O}_{1 / \kappa}\left(D_{r}\right)$ and $|z|<\varepsilon<r$, where $\mathbf{E}_{1 / \kappa}(t):=\sum_{n=0}^{\infty} \frac{t^{n}}{\Gamma(1+n / \kappa)}$ is the Mittag-Leffler function of order $1 / \kappa, \theta \in\left(-\arg w-\frac{\pi}{2},-\arg w+\frac{\pi}{2}\right)$ and $\oint_{|w|=\varepsilon}^{\kappa}$ means that we integrate $\kappa$ times along the positively oriented circle of radius $\varepsilon$. Here the integration in the inner integral is taken over the ray $\left\{e^{i \theta} r: r \geq\right.$ $\left.r_{0}\right\}$.

Under the above assumption, by a normalised formal solution $\hat{u}$ of (17) we mean such solution of (17), which is also a solution of the pseudodifferential equation $\widetilde{P}\left(\partial_{t}, \partial_{z}\right) \hat{u}=0$ (see [4, Definition 10]).

Since the principal part of the pseudodifferential operator $\widetilde{P}\left(\partial_{t}, \partial_{z}\right)$ with respect to $\partial_{t}$ is given by $\partial_{t}^{m}$, the Cauchy problem $(17)$ has a unique normalised formal power series solution $\hat{u} \in \mathcal{O}(D)[[t]]$.

Next, we reduce the Cauchy problem (17) of a general linear partial differential equation with constant coefficients to a family of the Cauchy problems of simple pseudodifferential equations. Namely we have 
HYPERASYMPTOTIC SOLUTIONS FOR CERTAIN PARTIAL DIFFERENTIAL EQUATIONS 11

Proposition 1 ([5, Theorem 1]). Let $\hat{u}$ be the normalised formal solution of (17). Then $\hat{u}=\sum_{\alpha=1}^{l} \sum_{\beta=1}^{m_{\alpha}} \hat{u}_{\alpha \beta}$ with $\hat{u}_{\alpha \beta}$ being a formal solution of a simple pseudodifferential equation

$$
\left\{\begin{array}{l}
\left(\partial_{t}-\lambda_{\alpha}\left(\partial_{z}\right)\right)^{\beta} \hat{u}_{\alpha \beta}=0 \\
\partial_{t}^{j} \hat{u}_{\alpha \beta}(0, z)=0(j=0, \ldots, \beta-2) \\
\partial_{t}^{\beta-1} \hat{u}_{\alpha \beta}(0, z)=\lambda_{\alpha}^{\beta-1}\left(\partial_{z}\right) \varphi_{\alpha \beta}(z),
\end{array}\right.
$$

where $\varphi_{\alpha \beta}(z):=\sum_{j=0}^{m-1} d_{\alpha \beta j}\left(\partial_{z}\right) \varphi_{j}(z) \in \mathcal{O}_{1 / \kappa}(D)$ and $d_{\alpha \beta j}(\zeta)$ are some holomorphic functions of the variable $\xi=\zeta^{1 / \kappa}$ and of polynomial growth.

Moreover, if $q_{\alpha}$ is a pole order of $\lambda_{\alpha}(\zeta)$ and $\bar{q}_{\alpha}=\max \left\{0, q_{\alpha}\right\}$, then a formal solution $\hat{u}_{\alpha \beta}$ is a Gevrey series of order $\bar{q}_{\alpha}-1$ with respect to $t$.

For this reason we will study the following simple pseudodifferential equation

$$
\left\{\begin{array}{l}
\left(\partial_{t}-\lambda\left(\partial_{z}\right)\right)^{\beta} u=0 \\
\partial_{t}^{j} u(0, z)=0(j=0, \ldots, \beta-2) \\
\partial_{t}^{\beta-1} u(0, z)=\lambda^{\beta-1}\left(\partial_{z}\right) \varphi(z) \in \mathcal{O}_{1 / \kappa}(D)
\end{array}\right.
$$

where $\lambda(\zeta) \sim \lambda \zeta^{q}$ for some $q \in \mathbb{Q}, q>1$. So we assume that $q=\mu / \nu$ for some relatively prime $\mu, \nu \in \mathbb{N}, \mu>\nu$.

3.3. Summable solutions of simple pseudodifferential equations. We have the following representation of summable solutions of (21).

Theorem 2. Let $k:=(q-1)^{-1}$ and $d \in \mathbb{R}$. Suppose that $\hat{u}(t, z)$ is a unique formal power series solution of the Cauchy problem (21) and

$$
\varphi(z) \in \mathcal{O}_{1 / \kappa}^{q k}\left(D \cup \bigcup_{l=0}^{q \kappa-1} S_{(d+\arg \lambda+2 l \pi) / q}\right) .
$$

Then $\hat{u}(t, z)$ is $k$-summable in the direction $d$ and its $k$-sum is given by

$$
u(t, z)=u^{d}(t, z)=\frac{1}{t^{1 / q}} \int_{e^{\frac{i d}{q}}} v\left(s^{q}, z\right) C_{q}\left(s / t^{1 / q}\right) d s,
$$

where

$$
v(t, z):=\hat{\mathcal{B}}_{1 / k} \hat{u}(t, z)=\hat{\mathcal{B}}_{1 / k}\left(\sum_{n=0}^{\infty} \frac{u_{n}(z)}{n !} t^{n}\right)=\sum_{n=0}^{\infty} \frac{u_{n}(z)}{\Gamma(1+q n)} t^{n} \in \mathcal{O}_{1,1 / \kappa}^{q}\left(\left(D \cup S_{d}\right) \times D\right)
$$

has the integral representation

$$
v(t, z)=\frac{t^{\beta-1}}{(\beta-1) !} \partial_{t}^{\beta-1} \frac{1}{2 \kappa \pi i} \oint_{|w|=\varepsilon}^{\kappa} \varphi(w) \int_{e^{i \theta} r_{0}}^{e^{i \theta} \infty} \mathbf{E}_{q}(t \lambda(\zeta)) \mathbf{E}_{1 / \kappa}\left(\zeta^{1 / \kappa} z^{1 / \kappa}\right) e^{-\zeta w} d \zeta d w
$$


with $\theta \in\left(-\arg w-\frac{\pi}{2},-\arg w+\frac{\pi}{2}\right)$. Moreover, if $\varphi \in \mathcal{O}_{\mathcal{A}}(\mathbb{C} \backslash H)$ and $z \in D_{\tilde{\varepsilon}}$ for some $\tilde{\varepsilon}>0$ then the function $t \mapsto v(t, z)$ is holomorphic for $|t|<\frac{(r-\tilde{\varepsilon})^{q}}{|\lambda|}$, where $r:=\min _{1 \leq i \leq K}\left|a_{i 1}\right|$.

Proof. First, observe that by Proposition 1 we get $\hat{u}(t, z) \in \mathcal{O}_{1 / \kappa}(D)[[t]]_{q-1}$. Moreover, by [5, Proposition 7] the function $v(t, z)=\hat{\mathcal{B}}_{1 / k} \hat{u}(t, z) \in \mathcal{O}_{1,1 / \kappa}\left(D^{2}\right)$ satisfies the moment partial differential equation

$$
\left\{\begin{array}{l}
\left(\partial_{t, \Gamma_{q}}-\lambda\left(\partial_{z}\right)\right)^{\beta} v=0 \\
\partial_{t, \Gamma_{q}}^{j} v(0, z)=0(j=0, \ldots, \beta-2) \\
\partial_{t, \Gamma_{q}}^{\beta-1} v(0, z)=\lambda^{\beta-1}\left(\partial_{z}\right) \varphi(z) \in \mathcal{O}_{1 / \kappa}(D),
\end{array}\right.
$$

where $\Gamma_{q}$ is a moment function defined by $\Gamma_{q}(n):=\Gamma(1+n q)$ for $n \in \mathbb{N}_{0}$ and $\partial_{t, \Gamma_{q}}$ is so called $\Gamma_{q}$-moment differential operator defined by (see [5, Definition 12])

$$
\partial_{t, \Gamma_{q}}\left(\sum_{n=0}^{\infty} \frac{a_{n}(z)}{\Gamma_{q}(n)} t^{n}\right):=\sum_{n=0}^{\infty} \frac{a_{n+1}(z)}{\Gamma_{q}(n)} t^{n} .
$$

Hence by [5, Lemma 3] with $m_{1}(n)=\Gamma_{q}(n)$ and $m_{2}(n)=\Gamma(1+n)$ we get the integral representation (25) of $v(t, z)$.

Since $\varphi(z)$ satisfies $\sqrt[22)]{ }$, by [5, Lemma 4] we conclude that $v(t, z) \in \mathcal{O}_{1,1 / \kappa}^{q}((D \cup$ $\left.\left.S_{d}\right) \times D\right)$. So, the function $u^{d}(t, z):=\mathcal{L}_{k, d} v(t, z)$ is well-defined and is given by (23).

Since the Mittag-Leffler function is the entire function satisfying the estimation $\left|\mathbf{E}_{q}(z)\right| \leq C e^{|z|^{1 / q}}$ (see [1, Appendix B.4]), the integrand in the inner integral in 25$]$ is estimated for $|z|<\widetilde{\varepsilon}$ by

$$
\left|\mathbf{E}_{q}(t \lambda(\zeta)) \mathbf{E}_{1 / \kappa}\left(\zeta^{1 / \kappa} z^{1 / \kappa}\right) e^{-\zeta w}\right| \leq \tilde{C} e^{|\zeta|\left(|\lambda|^{1 / q}|t|^{1 / q}-|w|+\widetilde{\varepsilon}\right)}
$$

as $\zeta \rightarrow \infty, \arg \zeta=\theta=-\arg w$. By the hypothesis $\varphi(w)$ is holomorphic for $|w|<r$, so we may deform the path of integration in the outer integral in 25 from $|w|=\varepsilon$ to $|w|=\tilde{r}$ for any $\tilde{r}<r$. It means that the inner integral in (25) is convergent for any $t$ satisfying $|t|<\frac{(r-\widetilde{\varepsilon})^{q}}{|\lambda|}$ and the function $t \mapsto v(t, z)$ is holomorphic for such $t$.

\subsection{Hyperasymptotic expansion of solution of simple pseudodifferential equa-}

tions. Using the change of variables to (23), as in the case of the heat equation we obtain

$$
u^{\theta}(t, z)=\frac{1}{q t^{1 / q}} \int_{0}^{e^{i \theta} \infty} \frac{1}{s^{1-\frac{1}{q}}} v(s, z) C_{q}\left((s / t)^{1 / q}\right) d s,
$$

so as $t \rightarrow 0, \arg t=\theta$ we conclude that

$$
u^{\theta}(t, z)=\frac{1}{q|t|^{1 / q}} \int_{0}^{\infty} \frac{1}{s^{1-\frac{1}{q}}} v\left(s e^{i \theta}, z\right) C_{q}\left((s /|t|)^{1 / q}\right) d s
$$


for any $\theta$ different from the Stokes lines, i.e. $\theta \neq q \lambda_{i}-\arg \lambda \bmod 2 \pi$ for $i=1, \ldots, K$.

Now we are ready to repeat the construction of the hyperasymptotic expansion for the heat equation under condition that $\varphi \in \mathcal{O}_{\mathcal{A}}^{k q}(\mathbb{C} \backslash H)$ (i.e. $\varphi \in \mathcal{O}_{\mathcal{A}}(\mathbb{C} \backslash H)$ and $\varphi(z)$ has the exponential growth of order $k q$ as $z \rightarrow \infty, z \in \mathbb{C} \backslash H)$. We also assume that the direction $\theta$ is separated from the Stokes lines, i.e. that

$\theta \in[0,2 \pi) \backslash \bigcup_{i=1}^{K}\left(q \lambda_{i}-\arg \lambda-\delta, q \lambda_{i}-\arg \lambda+\delta\right) \quad \bmod 2 \pi$ for fixed $\delta>0$.

We put $f_{0}(s, z):=v\left(s e^{i \theta}, z\right), r:=\min _{1 \leq i \leq K}\left|a_{i 1}\right|-\widetilde{\varepsilon}$ and

$$
\Omega_{0}(0, s):=\partial\left(\left\{w \in \mathbb{C}:|w| \leq \frac{r^{q}}{|\lambda|}-\varepsilon\right\} \cup\left\{w \in \mathbb{C}:|w-s| \leq \frac{\varepsilon}{2}\right\}\right)
$$

for some $\varepsilon \in\left(0, \frac{r^{q}}{2|\lambda|}\right)$.

Observe that by Theorem 2 for any $z \in D_{\widetilde{\varepsilon}}$ the function $w \mapsto f_{0}(w, z)$ is holomorphic in the domain bounded by $\Omega_{0}(0, s)$. By [4, Lemma 2]

$$
u(t, z)=\sum_{j=\beta-1}^{N_{0}-1}\left(\begin{array}{c}
j \\
\beta-1
\end{array}\right) \frac{\lambda^{j}\left(\partial_{z}\right) \varphi(z)}{j !} t^{j}+R_{N_{0}}(t, z) .
$$

Moreover, as in the case of the heat equation

$$
R_{N_{0}}(t, z)=\frac{1}{q|t|^{1 / q}} \int_{0}^{\infty} s^{N_{0}-1+\frac{1}{q}} C_{q}\left((s /|t|)^{1 / q}\right) f_{1}(s, z) d s,
$$

where $f_{1}(s, z)$ is defined as in (7).

By (24) there exist positive constants $A^{\prime}$ and $B^{\prime}$ such that

$$
\left|f_{0}(w, z)\right| \leq A^{\prime} e^{B^{\prime}|s|^{k}} \quad \text { for any } \quad w \in \Omega_{0}(0, s) .
$$

Hence

$$
\begin{aligned}
\left|f_{1}(s, z)\right| \leq \frac{1}{2 \pi} \int_{\Omega_{0}(0, s)} \frac{2 A^{\prime} e^{B^{\prime}|s|^{k}}}{\varepsilon\left(\frac{r^{q}}{|\lambda|}-\varepsilon\right)^{N_{0}}} d|w| & \leq \frac{2 A^{\prime} e^{B^{\prime}|s|^{k}}}{\varepsilon\left(\frac{r^{q}}{|\lambda|}-\varepsilon\right)^{N_{0}}}\left(\frac{r^{q}}{|\lambda|}-\varepsilon+\frac{\varepsilon}{2}\right) \\
& \leq \frac{2 A^{\prime} r^{q} e^{B^{\prime}|s|^{k}}}{|\lambda| \varepsilon\left(\frac{r^{q}}{|\lambda|}-\varepsilon\right)^{N_{0}}}=\frac{A_{0}^{\prime} e^{B^{\prime}|s|^{k}}}{\left(\frac{r^{q}}{|\lambda|}-\varepsilon\right)^{N_{0}}}
\end{aligned}
$$

where $A_{0}^{\prime}:=\frac{2 A^{\prime} r^{q}}{|\lambda| \varepsilon}$.

Moreover, by the properties of the Ecalle kernel (see [3, Lemma 6]) we may estimate

$$
\left|C_{q}(\tau)\right| \leq C e^{-\left(\tau^{k+1} / c_{q}\right)} \quad \text { with } \quad c_{q}=(k+1)^{k+1} k^{-k} .
$$

So

$$
\left|R_{N_{0}}(t, z)\right| \leq \frac{A_{0}^{\prime}}{q|t|^{1 / q}} \int_{0}^{\infty} s^{N_{0}-1+\frac{1}{q}} e^{-s^{k}\left(\frac{1}{c_{q}|t|^{k}}-B^{\prime}\right)}\left(\frac{r^{q}}{|\lambda|}-\varepsilon\right)^{-N_{0}} d s .
$$


Similarly to the heat equation case we conclude that the integrand of (27) has a maximum at certain point $s=\sigma_{1}$ satisfying $N_{0}=k \sigma_{1}^{k}\left(\frac{1}{c_{q}|t|^{k}}-B^{\prime}\right)+1-\frac{1}{q}$. Now, the minimum with respect to $\sigma_{1}$ is given at $\sigma_{1}=\frac{r^{q}}{|\lambda|}-\varepsilon$. Hence we take $N_{0}:=\left\lfloor k\left(\frac{r^{q}}{|\lambda|}-\varepsilon\right)^{k}\left(\frac{1}{c_{q}|t|^{k}}-B^{\prime}\right)+1-\frac{1}{q}\right\rfloor$ and $\sigma_{1}:=\left(\frac{N_{0}+\frac{1}{q}-1}{k\left(\frac{1}{c_{q}|t|^{k}}-B^{\prime}\right)}\right)^{1 / k} \cdot$ Observe that $\sigma_{1} \leq \frac{r^{q}}{|\lambda|}-\varepsilon$. So we are able to use the Laplace method and to conclude that

$$
\left|R_{N_{0}}(t, z)\right| \sim O\left(\frac{e^{-\sigma_{1}^{k}\left(\frac{1}{c_{q}|t|^{k}}-B^{\prime}\right)}}{|t|^{1 / q} \sqrt{\frac{1}{c_{q}|t|^{k}}-B^{\prime}}}\right) \quad \text { for } \quad t \rightarrow 0, \arg t=\theta, z \in D_{\tilde{\varepsilon}} .
$$

Next, we construct the $n$-level hyperasymptotic expansion as for the heat equation. The remainder obtained in the $n$-level hyperasymptotic expansion is of the form

$R_{N_{n}}(t, z)=\frac{1}{q|t|^{1 / q}} \int_{0}^{\infty} C_{q}\left(\left(\frac{s}{|t|}\right)^{\frac{1}{q}}\right) s^{N_{0}-1+\frac{1}{q}}\left(s-\sigma_{1}\right)^{N_{1}} \cdots\left(s-\sigma_{n}\right)^{N_{n}} f_{n+1}(s, z) d s$,

where

Here we take

$$
f_{n+1}(s, z):=\frac{1}{2 \pi i} \int_{\Omega_{n}\left(\sigma_{n}, s\right)} \frac{f_{n}(w, z)}{\left(w-\sigma_{n}\right)^{N_{n}}(w-s)} d w .
$$

$\Omega_{n}\left(\sigma_{n}, s\right):=\partial\left(\left\{w \in \mathbb{C}:\left|w-\sigma_{n}\right| \leq d\left(\sigma_{n}, \theta\right)-\rho_{n} \varepsilon\right\} \cup\left\{w \in \mathbb{C}:|w-s| \leq 2^{-n-1} \varepsilon\right\}\right)$ with $d\left(\sigma_{n}, \theta\right):=\inf _{z \in D_{\tilde{\varepsilon}}} \inf _{\zeta \in H}\left|\sigma_{n}-e^{-i \theta} \lambda(z-\zeta)^{q}\right|$ and $\rho_{n}:=2-2^{-n}$.

Using the same algorithm as in the case of the heat equation, we can estimate $R_{N_{n}}(t, z)$ as follows

$$
\begin{array}{r}
\left|R_{N_{n}}(t, z)\right| \leq \frac{A_{n}^{\prime}}{q|t| 1 / q} \int_{0}^{\infty} e^{-s^{k}\left(\frac{1}{c_{q}|t|^{k}}-B^{\prime}\right)} \frac{s^{N_{0}-1+\frac{1}{q}}}{\left(\frac{r^{q}}{|\lambda|}-\varepsilon\right)^{N_{0}}} \cdot \frac{\left|s-\sigma_{1}\right|^{N_{1}}}{\left(d\left(\sigma_{1}, \theta\right)-\rho_{1} \varepsilon\right)^{N_{1}}} \\
\cdots \frac{\left|s-\sigma_{n}\right|^{N_{n}}}{\left(d\left(\sigma_{n}, \theta\right)-\rho_{n} \varepsilon\right)^{N_{n}}} d s
\end{array}
$$

for a certain constant $A_{n}^{\prime}$.

Let us observe that the integrand on the right-hand side of $(28)$ has $n+1$ maxima in points $s_{1}, \ldots, s_{n+1}$ such that $s_{1}<\sigma_{1}<s_{2}<\ldots<\sigma_{n}<s_{n+1}$ and all $s_{j}$ satisfy the condition:

$$
k s_{j}^{k-1}\left(\frac{1}{c_{q}|t|^{k}}-B^{\prime}\right)=\frac{N_{0}-1+\frac{1}{q}}{s_{j}}+\frac{N_{1}}{s_{j}-\sigma_{1}}+\ldots+\frac{N_{n}}{s_{j}-\sigma_{n}} .
$$

From this, as in the case of the heat equation, we conclude that $s_{j}$ are decreasing functions of $N_{n}$ for $1 \leq j \leq n$ and $s_{n+1}$ increases to infinity as $N_{n} \rightarrow \infty$. Similarly, the value of the integrand in $(29)$ in the points $s_{j}, 1 \leq j \leq n$, decreases 
with respect to $N_{n}$. Moreover, this value in the point $s_{n+1}$ decreases with respect to $N_{n}$ for $s_{n+1}<\sigma_{n}+d\left(\sigma_{n}, \theta\right)-\rho_{n} \varepsilon$ and increases when $s_{n+1}>\sigma_{n}+d\left(\sigma_{n}, \theta\right)-$ $\rho_{n} \varepsilon$.

Hence, as in the case of the heat equation there exists $N_{n} \in \mathbb{N}$ and $s_{n+1}$ for which the integrand reaches its minimal value. We denote such $s_{n+1}$ by $\sigma_{n+1}$.

Again, using the Laplace method we obtain the estimation of $R_{N_{n}}$

$$
\left|R_{N_{n}}(t, z)\right| \sim O\left(\frac{e^{-\tilde{\eta}_{n}^{k}\left(\frac{1}{c_{q}|t|^{k}}-B^{\prime}\right)}}{|t|^{1 / q} \sqrt{\frac{1}{c_{q}|t|^{k}}-B^{\prime}}}\right) \quad \text { for } \quad t \rightarrow 0, \arg t=\theta, z \in D_{\tilde{\varepsilon}}
$$

where, as previously $\tilde{\eta}_{0}=\sigma_{1} \sim \frac{r^{q}}{|\lambda|}<\tilde{\eta}_{1}<\tilde{\eta}_{2}<\tilde{\eta}_{3}<\ldots$ is some increasing sequence of positive numbers (see [7] and [8]).

To find the $(n+1)$-level hyperasymptotic expansion we expand the function $s \mapsto f_{n+1}(s, z)$ around the point $\sigma_{n+1}$ as in (14), which, after substituting it in (28), gives us the $(n+1)$-level expansion

$$
\begin{array}{r}
R_{N_{n}}(t, z)=\frac{1}{q|t|^{1 / q}} \sum_{j=0}^{N_{n+1}-1} b_{n+1, j}(z) \int_{0}^{\infty} C_{q}\left(\left(\frac{s}{|t|}\right)^{\frac{1}{q}}\right) s^{N_{0}-1+\frac{1}{q}}\left(s-\sigma_{1}\right)^{N_{1}} \\
\cdots\left(s-\sigma_{n}\right)^{N_{n}}\left(s-\sigma_{n+1}\right)^{j} d s+R_{N_{n+1}}(t, z) .
\end{array}
$$

Since the Laplace transform $\mathcal{L}_{k, d}$ is inverse to $k$-Borel transform $\hat{\mathcal{B}}_{k}$, we conclude that $\mathcal{L}_{k, d}\left(t^{l}\right)=\frac{\Gamma(1+q l)}{l !} t^{l}$ for $l=0,1, \ldots$ It means that

$$
\frac{1}{q|t|^{1 / q}} \int_{0}^{\infty} \frac{C_{q}\left(\left(\frac{s}{|t|}\right)^{\frac{1}{q}}\right)}{s^{1-\frac{1}{q}}} s^{l} d s=\frac{\Gamma(1+q l)}{l !}|t|^{l}=\frac{\Gamma(1+q l)}{l !} e^{-i \theta l} t^{l},
$$

and using (15) we get

$$
R_{N_{n}}(t, z)=\sum_{j=0}^{N_{n+1}-1} b_{n+1, j}(z) \sum_{l=0}^{N_{0}+\cdots+N_{n}+j} a_{n, j, l} \frac{\Gamma(1+q l)}{l !} e^{-i \theta l} t^{l}+R_{N_{n+1}}(t, z) .
$$

Hence, as in the case of the heat equation, we conclude that the hyperasymptotic expansion of $u$ takes also the form $(16)$ for some functions $\psi_{l}(z)$.

Finally, similarly to the heat equation, we get as the conclusion

Theorem 3 (Hyperasymptotic expansion for the simple equation). For every $n \in$ $\mathbb{N}$ the solution of the equation $(21)$ with $\varphi \in \mathcal{O}_{\mathcal{A}}^{k q}(\mathbb{C} \backslash H)$ has the hyperasymptotic expansion as t tends to zero in a direction $\theta \in[0,2 \pi) \backslash \bigcup_{i=1}^{K}\left(q \lambda_{i}-\arg \lambda-\delta, q \lambda_{i}-\right.$ 
$\arg \lambda+\delta) \bmod 2 \pi$, which has the form

$$
\begin{array}{r}
u^{\theta}(t, z)=\sum_{j=\beta-1}^{N_{0}-1}\left(\begin{array}{c}
j \\
\beta-1
\end{array}\right) \frac{\lambda^{j}\left(\partial_{z}\right) \varphi(z)}{j !} t^{j}+\sum_{m=1}^{n} \sum_{j=0}^{N_{m}-1} \frac{b_{m, j}(z)}{|t|^{1 / q}} \int_{0}^{\infty} \frac{1}{q s^{1-\frac{1}{q}}} \\
\cdot C_{q}\left((s /|t|)^{1 / q}\right) s^{N_{0}}\left(s-\sigma_{1}\right)^{N_{1}} \cdots\left(s-\sigma_{m-1}\right)^{N_{m-1}}\left(s-\sigma_{m}\right)^{j} d s+R_{N_{n}}(t, z) \\
=\sum_{l=0}^{N_{0}+\cdots+N_{n}-1} \psi_{l}(z) t^{l}+R_{N_{n}}(t, z),
\end{array}
$$

where

$$
\begin{gathered}
b_{m, j}(z)=\left.\frac{1}{j !} \frac{\partial^{j}}{\partial s^{j}} f_{m}(s, z)\right|_{s=\sigma_{m}} \\
R_{N_{n}}(t, z)=\frac{1}{|t|^{1 / q}} \int_{0}^{\infty} \frac{1}{q s^{1-\frac{1}{q}}} C_{q}\left(\left(\frac{s}{|t|}\right)^{\frac{1}{q}}\right) s^{N_{0}}\left(s-\sigma_{1}\right)^{N_{1}} \cdots\left(s-\sigma_{n}\right)^{N_{n}} f_{n+1}(s, z) d s, \\
f_{m}(s, z)=\frac{1}{(2 \pi i)^{m}} \int_{\Omega_{m-1}\left(\sigma_{m-1}, s\right)} \int_{\Omega_{m-2}\left(\sigma_{m-2}, x_{m-1}\right)} \cdots \int_{\Omega_{1}\left(\sigma_{1}, x_{2}\right)} \int_{\Omega_{0}\left(0, x_{1}\right)} \\
\frac{v\left(x_{0} e^{i \theta}, z\right) d x_{0} \ldots d x_{m-1}}{x_{0}^{N_{0}}\left[\prod_{k=1}^{m-1}\left(x_{k}-\sigma_{k}\right)^{N_{k}}\left(x_{k-1}-x_{k}\right)\right]\left(x_{m-1}-s\right)}
\end{gathered}
$$

and $v(s, z)$ is defined by (25).

$$
\text { Moreover } R_{N_{n}}(t, z) \sim O\left(\frac{e^{-\tilde{\eta}_{n}^{k}\left(\frac{1}{c_{q} \mid t k^{k}}-B^{\prime}\right)}}{|t|^{1 / q} \sqrt{\frac{1}{c_{q}|t|^{k}}-B^{\prime}}}\right) \text { as } t \rightarrow 0, \arg t=\theta, z \in D_{\tilde{\varepsilon}}
$$

for some sequence of positive numbers $\tilde{\eta}_{0}=\sigma_{1} \sim \frac{r^{q}}{|\lambda|}<\tilde{\eta}_{1}<\tilde{\eta}_{2}<\tilde{\eta}_{3}<\ldots$

Acknowledgements. The authors would like to thank the anonymous referee for valuable comments, suggestions, and especially for the indication of the form of hyperasymptotic expansion of the solution $u(t, z)$ presented in (16).

\section{REFERENCES}

[1] W. Balser, Formal power series and linear systems of meromorphic ordinary differential equations, Springer-Verlag, New York, 2000.

[2] M. V. Berry, C. J. Howls, Hyperasymptotics, Proc. R. Soc. Lond. Ser. A 430 (1990), 653668.

[3] J. Martinet, J-P Ramis, Elementary acceleration and multisummability. I, Annales de 1'I. H. P., section A, 54 (1991), 331-401.

[4] S. Michalik, Analytic solutions of moment partial differential equations with constant coefficients, Funkcial. Ekvac., 56 (2013), 19-50.

[5] S. Michalik, Summability of formal solutions of linear partial differential equations with divergent initial data, J. Math. Anal. Appl. 406 (2013), 243-260.

[6] S. Michalik, B. Podhajecka, The Stokes phenomenon for certain partial differential equations with meromorphic initial data, Asymptot. Anal. 99, 2016, 163-182.

[7] A.B. Olde Daalhuis, Hyperasymptotic expansions of confluent hypergeometric functions, IMA J. Appl. Math 49 (1992), 203-216. 
[8] A.B. Olde Daalhuis, Hyperasymptotics and the Stokes' phenomenon, Proc. Roy. Soc. Edinburgh Sect. A 123 (1993), 731-743.

[9] A.B. Olde Daalhuis, Hyperasymptotic solutions of higher order linear differential equations with a singularity of rank one, Proc. R. Soc. Lond. Ser. A 454 (1998), 1-29.

[10] A.B. Olde Daalhuis, Hyperasymptotics for nonlinear ODEs I. A Riccati equation, Proc. R. Soc. Lond. Ser. A 461 (2005), 2503-2520.

[11] A.B. Olde Daalhuis, Hyperasymptotics for nonlinear ODEs II. The first Painlevé equation and a second-order Riccati equation, Proc. R. Soc. Lond. Ser. A 461 (2005), 3005-3021.

[12] F. W. J. Olver, Asymptotics and Special Functions, Academic Press, New York, 1974.

Faculty of Mathematics and Natural Sciences, College of Science, CardiNAL STEFAn WySZYŃSKi UNIVERSITY, WÓYCICKIEGo 1/3, 01-938 WARSZAWA, POLAND

E-mail address: s.michalikeuksw.edu.pl $U R L:$ http://www.impan.pl/ slawek

Faculty of Mathematics and Natural Sciences, College of Science, CardiNAL STEFAN WySZYŃSKi UNIVERSITY, WÓYCICKIEGO 1/3, 01-938 WARSZAWA, PolAND

E-mail address: m. suwinska@op.pl 